\title{
Identificazione e resistenza agli antibiotici di ceppi ben caratterizzati analizzati in laboratori liguri
}

\author{
Marina Casini Lemmi', Giovanna Dho $^{3}$, Graziana Manno², Anna Marchese, \\ Eugenio A Debbia ${ }^{3}$ per AMCLI LIGURIA
}

\author{
'Laboratorio Analisi, Ospedali Galliera \\ ${ }^{2}$ Laboratorio Ricerca e Diagnostica Infettivologica Dipartimento di Pediatria, Istituto G. Gaslini, \\ ${ }^{3}$ Sez. Microbiologia DISCAT, Università di Genova.
}

\begin{abstract}
Identification and resistance to antibiotics of well-characterized strains analyzed in Ligurian laboratories
\end{abstract}

Key words: Antibiotic-resistance, quality control, well-characterized strains

\section{SUMMARY}

During the year 2002, 19 Clinical Microbiology Laboratories including 3 private centres, distributed in Ligurian and 2 in South Piemonte area have studied 26 strains carrying well-characterized antibiotic-resistant traits. Only 7 laboratories analysed the complete collection of 26 isolates and 12 sites studied at least 20 strains. Overall $85.4 \%$ of the microrganism identifications and $82.8 \%$ of the antibiotic susceptibility tests were correct. Gramnegative bacteria were more easily identified than the Gram-positive counterpart being $E$. faecium the most difficult pathogen to identify. Discrepancies were also observed with the antibiotic susceptibility tests. A significant number of tests, $22.6 \%$ of the identifications and $29.9 \%$ of the antibiotic susceptibility determinations were not carried out due to local economic and structural problems. Many of the pathogens are rarely encountered by Clinical Microbiology laboratories and sometime are difficult to detect, however, a large number of the centres was able to identify correctly these particular pathogens and their antibiotypes.

\section{INTRODUZIONE}

L'insorgenza di microrganismi resistenti agli antibiotici è un fenomeno che ha assunto dimensioni mondiali pur non coinvolgendo in eguale misura tutte le specie batteriche e le diverse classi di agenti antimicrobici $(6,9,12,22$, 23, 29).

Usualmente l'incidenza con cui vengono isolati germi resistenti è dipendente dalla pressione selettiva esercitata qualitativamente e quantitativamente dagli antibiotici. A causa delle diverse abitudini terapeutiche delle comunità mediche l'impiego dei farmaci antimicrobici è variabile non solo da Paese a Paese ma anche da zona a zona nell'ambito della stessa Nazione. Poiché l'insensibilità in vitro ad un antibiotico può correlare con un possibile fallimento terapeutico (1), si promuovono periodicamente indagini multicentriche nazionali e internazionali con lo scopo di acquisire una corretta informazione sulla diffusione delle resistenze agli antibiotici $(10,11,21,23,27,28,29)$. Il laboratorio di Microbiologia Clinica spesso oberato da un pesante lavoro routinario, deve garantire la perfetta esecuzione dell'esame batteriologico attraverso continui aggiornamenti della letteratura referenziata e mediante l'inserimento nei saggi di routine di ceppi per $\mathrm{i}$ controlli di qualità $(11,20)$; infatti l'esame batteriologico deve prevedere, specie in ambiente nosocomiale, l'accertamento delle resistenze agli antibiotici sui ceppi isolati, con particolare attenzione a quei caratteri di insensibilità non sempre chiaramente evidenti nei saggi routinari, ma spesso più temibili poiché se non identificati potrebbero portare ad un fallimento terapeutico $(21,27,28)$.

Questo studio riporta i risultati di una indagine condotta durante il periodo giugno-settembre 2002, dal Gruppo Scientifico della Sezione Ligure dell'AMCLI volta ad accertare la "proficiency" di laboratorio di microbiologia a livello regionale. Lo studio è stato indirizzato ad accertare, in prima istanza la corretta identificazione a livello di specie di patogeni Gram positivi e Gram negativi e, in secondo luogo, la corretta rivelazione dei fenotipi di resistenza in ceppi ben caratterizzati veicolanti vari tipi e livelli di resistenza agli agenti antibatterici. Ad ogni centro che ha aderito all'iniziativa è stata inviata da parte del laboratorio di riferimento una collezione di ceppi con caratteristiche note. Inoltre sono stati forniti una serie di stipiti ATCC per il controllo interno di qualità. Ad ogni laboratorio è stato richiesto di identificare i microrganismi ed evidenziare le eventuali resistenze ai farmaci antimicrobici seguendo le metodiche abitualmente in uso presso 
il centro. I risultati sono stati inviati al laboratorio di riferimento ove il Gruppo di Studio promotore dell'indagine li ha esaminati.

\section{MATERIALI E METODI \\ Centri Partecipanti allo studio}

24 Laboratori di Microbiologia Clinica hanno aderito all'iniziativa proposta; l'elenco è riportato nella tabella 1 . I centri che non hanno inviato $i$ risultati sono stati esclusi dallo studio.

\section{Microrganismi}

In questo studio sono stati utilizzati ceppi appartenenti alla collezione dell'Istituto di Microbiologia dell'Università di Genova, le cui caratteristiche e origine sono riassunte nella tabella 2. I ceppi conservati in glicerolo a $-80^{\circ} \mathrm{C}$ sono stati seminati su agar nutriente, incubati $24-$

Tabella I. Elenco dei centri e dei partecipanti allo studio

\begin{tabular}{lll}
\hline LOCALITÀ & LABORATORIO & PARTECIPANTI \\
\hline Genova- Rivarolo & Ospedale Celesta & Caprifoglio C. \\
\hline Genova & Istituto G. Gaslini, & Barretta M.A., Pescetto L., Bandettini R. \\
\hline Ovada (AL) & Ospedale di Ovada ASL 22 & Mazzarello M. G., Torriglia A. M., Ferrari M. \\
\hline Genova & Ospedale Evangelico & Intra E., Serra D. \\
\hline Genova & DiSCAT, Sezione di Microbiologia & Balistreri M. \\
\hline Genova & Istituto G. Gaslini Malattie Infettive & Manno G. \\
\hline Tortona (AL) & Ospedale di Tortona ASL 20 & Salerno A. \\
\hline Genova -Voltri & Ospedale S. Carlo & Illiberi O., Galleano A. \\
\hline La Spezia & Ospedale di La Spezia & Fregoso F. \\
\hline Genova & Ospedale Galliera & Casini-Lemmi M., Usiglio D. \\
\hline Sarzana (SP) & Ospedale S. Bartolomeo & Battola E., Benini G. \\
\hline Genova-Sampierdarena & Ospedale Villa Scassi & Capuzzo R., Riva R., Carcheri M. \\
\hline Savona & Ospedale S.Paolo & Bona R. \\
\hline Imperia & Ospedale di Imperia & Diotto I. \\
\hline Pietra L. (SV) & Ospedale S.Corona & Santoriello L. \\
\hline SanRemo (IM) & Ospedale di Sanremo,AsI I Imperiese & Vassallo M., Dusi P.A. \\
\hline Genova & Lab srl & Aytano P. \\
\hline Genova & Laboratorio-Albaro Spa & Bonanni R., Clavarezza M.A. \\
\hline San Remo (IM) & Laboratorio Dini-Sccardi & Bertolini C. \\
\hline
\end{tabular}

Tabella 2. Elenco dei ceppi inviati ai vari centri e caratteristiche di resistenza principali

\begin{tabular}{|c|c|c|}
\hline Ceppo & Caratteristiche & Origine (referenze) \\
\hline 1 & S. aureus mecA costitutivo & Chung et al., 2000 (5) \\
\hline 2 & S. aureus mecA inducibile & Chung et al., 2000 (5) \\
\hline 3 & S. aureus penicillasi+ & Marchese et al., 1995 (16) \\
\hline 4 & S. haemolyticus teiR & Marchese et al., I997 (15) \\
\hline 5 & E. faecalis VanA & Boisivon et al., 1997 (2) \\
\hline 6 & E. faecium VanB & Boisivon et al., 1997 (2) \\
\hline 7 & E. gallinarum VanC & Boisivon et al., 1997 (2) \\
\hline 8 & E. faecalis amgR & Schito et al., $1990(24)$ \\
\hline 9 & E. faecium ampR & Marchese et al., 1997 (15) \\
\hline 10 & S. pneumoniae penS & Marchese et al., 2000 (I8) \\
\hline II & S. pneumoniae penl & Marchese et al., 2000 (18) \\
\hline 12 & S. pneumoniae penR & Marchese et al., 2000 (I8) \\
\hline 13 & S. pneumoniae eriR costitutivo & Marchese et al., 1999 (19) \\
\hline 14 & S. pneumoniae eriR fenotipo $M$ & Marchese et al., 1999 (19) \\
\hline 15 & S. pneumoniae eriR inducibile & Marchese et al., 1999 (19) \\
\hline 16 & S. pneumoniae eriS & Marchese et al., I999 (19) \\
\hline 17 & S. pyogenes eriS & Chelossi et al., 1997 e $1998(3,4)$ \\
\hline 18 & S. pyogenes eriR costitutivo & Chelossi et al., I997 e $1998(3,4)$ \\
\hline 19 & S. pyogenes eriR fenotipo $M$ & Chelossi et al., I997 e $1998(3,4)$ \\
\hline 20 & S. pyogenes eriR inducibile & Chelossi et al., I997 e $1998(3,4)$ \\
\hline 21 & E. coli ESBL & Marchese et al., I996 (I7) \\
\hline 22 & E. coli cipR & Livermore et al., 2002 (I3) \\
\hline 23 & E. coli ampR & Questo studio \\
\hline 24 & E. coli IRT & Livermore et al., 2002 (I3) \\
\hline 25 & P. aeruginosa imiR & Questo studio \\
\hline 26 & Salmonella spp ESBL & Livermore et al., 2002 (13) \\
\hline
\end{tabular}


$48 \mathrm{~h}$ a $35^{\circ} \mathrm{C}$ e quindi inseriti in tamponi con terreno di trasporto e inviati ai singoli centri. Ceppi di QC interno forniti: E. coli ATCC 25922, $E$. coli ATCC 35218 , per i saggi includenti un $\beta$ lattamico e un inibitore di $\beta$-lattamasi, $P$. aeruginosa ATCC 27853, S. aureus ATCC 25923 per le prove di diffusione da dischetto, $S$. aureus ATCC29213 per la valutazione delle MIC e $S$. aureus ATCC43300 come ceppo oxacillinoresistente (OXA-R), E. faecalis ATCC 29212 e E. faecalis ATCC 51299, per i saggi che includono il saggio rispettivamente della sensibilità e della resistenza agli aminoglicosidi e alla vancomicina, S. pneumoniae ATCC49619, H. influenzae ATCC 49247 e H. influenzae ATCC49766 per i saggi che includono i $\beta$-lattamici (20).

Metodologie di riferimento proposte per la caratterizzazione dei fenotipi di resistenza.

Sono di seguito riportate le metodologie utilizzate per la caratterizzazione dei fenotipi di resistenza. Queste sono state applicate dal Centro di riferimento e proposte ai Centri partecipanti.

Saggi di sensibilità agli antibiotici. Le minime concentrazioni inibenti (MIC) sono state determinate con il metodo della microdiluizione in brodo Mueller Hinton $(\mathrm{MH})$ contenente $\mathrm{Ca}++$ e $\mathrm{Mg}++$ o mediante sistema $\mathrm{E}$ test. Il metodo Kirby-Bauer della diffusione da dischetto è stato utilizzato in alcuni saggi in parallelo alla valutazione della MIC per confermare ulteriormente il fenotipo di resistenza. I saggi sono stati condotti e interpretati seguendo le linee guida suggerite dal National Committee for Clinical Laboratory Standards, 2002 (20).

B-lattamasi in $S$. aureus. La $\beta$-lattamasi (penicillinasi) in $S$. aureus è stata individuata mediante saggio della cefalosporina cromogena nitrocefin. E stato inoltre utilizzato il metodo della diffusione su agar impiegando dischetti di penicillina $(\mathrm{P})$ e amoxicillina-clavulanato (AUG), il ceppo evidenzia resistenza al capostipite dei $\beta$ lattamici $(\mathrm{P})$ e sensibilità all'associazione con l'inibitore dell'enzima (AUG). Il microrganismo presenta inoltre resistenza alla penicillina $(\mathrm{P}) \mathrm{e}$ sensibilità all'oxacillina (OXA-S).

Oxacillino-resistenza in $S$. aureus.

L'oxacillino-resistenza (OXA-R) inducibile (I) o costitutiva (C) in $S$. aureus è stata messa in evidenza utilizzando il saggio dell'agar screening su piastre di Mueller-Hinton agar con $6 \mathrm{mg} / \mathrm{L} \mathrm{di}$ oxacillina in duplicato, con e senza il $4 \%$ di $\mathrm{NaCl}$ e un inoculo di $5 \times 10^{5} \mathrm{CFU}$ per spot. La lettura è stata eseguita dopo 24 ore di incubazione. I ceppi sono stati mantenuti ad una temperatura non superiore ai $35.5^{\circ} \mathrm{C}$. In queste condizioni il ceppo inducibile è risultato sensibile al farmaco sul terreno privo di sale, mentre lo stipite costitutivo ha manifestato resistenza all'oxacillina su entrambi i terreni. S. epidermidis OXA-R, aggiunto come controllo in questi saggi, ma non incluso nello studio, è stato valutato mediante Kirby-Bauer con dischetto di oxacillina $(1 \mu \mathrm{g})$ su terreno $\mathrm{MH}$ non contenente $\mathrm{NaCl}$. Come è noto gli stafilococchi coagulasi-negativi (CNS) non si saggiano per questo carattere su terreno contenente sale. I breakpoint per CNS adottati sono stati $\mathrm{S} \geqslant 18 \mathrm{~mm}$ e $\mathrm{R} \leqslant 17 \mathrm{~mm}$. Lo stesso saggio con tecnica di KirbyBauer su $\mathrm{MH}$ privo di $\mathrm{NaCl}$ è stato utilizzato anche per $S$. aureus. In questo caso i breakpoint sono stati $\mathrm{S} \geqslant 13 \mathrm{~mm}$ e $\mathrm{R} \leqslant 10 \mathrm{~mm}$ (20).

Teicoplanino-resistenza in $S$. haemolyticus. S. haemolyticus resistente alla teicoplanina è stato identificato mediante Kirby-Bauer e successiva determinazione della minima concentrazione inibente adottando la tecnica della microdiluizione in brodo (20). Il saggio è stato letto dopo 24 ore di incubazione a $35.5^{\circ} \mathrm{C}$.

Resistenza ai glicopeptidi negli enterococchi. Per l'accertamento della refrattarietà ai glicopeptidi è stato inizialmente utilizzato il metodo Kirby-Bauer o lo screening su agar contenente $6 \mathrm{mg} / \mathrm{L}$ di vancomicina e un inoculo di $5 \times 10^{5} \mathrm{CFU}$ per spot. I fenotipi vanA, vanB e vanC sono stati distinti e confermati con la valutazione della MIC a teicoplanina e vancomicina mediante la tecnica della microdiluizione o E test (20). E. faecalis vanA risulta resistente a teicoplanina e vancomicina. $E$. faecalis vanB mostra sensibilità a teicoplanina e resistenza a vancomicina. vanC in E. gallinarum è sensibile a teicoplanina e resistente a basso livello $(\mathrm{MIC}=8-32 \mathrm{mg} / \mathrm{L})$ a vancomicina. Il test è stato letto dopo 24 ore di incubazione a $35.5^{\circ} \mathrm{C}$.

Alto livello di resistenza agli aminoglicosidi negli enterococchi. L'alto livello di resistenza agli aminoglicosidi negli enterococchi è stato valutato in piastre di BHIA contenenti $500 \mathrm{mg} / \mathrm{L}$ di gentamicina (gen) o $2000 \mathrm{mg} / \mathrm{L}$ di streptomicina (str). Per il controllo di qualità di questo test è stato impiegato E. faecalis ATCC 29212. Sono stati utilizzati inoculi di $10^{6} \mathrm{CFU}$ per spot. Dopo incubazione per 24 ore in aerobiosi le piastre sono state esaminate, la presenza di una o più colonie o un alone di crescita è stato considerato indice di resistenza. Quando il saggio con la streptomicina è risultato negativo la piastra è stata incubata nuovamente per altre 24 ore. In alternativa la resistenza agli aminoglicosidi è stata identificata con le apposite strisce di E test.

Ampicillina-resistenza in E. faecium.

L'insensibilità all'ampicillina in E. faecium è stata determinata mediante Kirby-Bauer e successiva conferma mediante valutazione della MIC in micrometodo.

Penicillino resistenza in Streptococcus pneumoniae. É stato utilizzato il dischetto di 
oxacillina $1 \mu \mathrm{g}$, con i limiti $\mathrm{S} \geqslant 20 \mathrm{~mm}$ e $\mathrm{R} \leqslant 19$ $\mathrm{mm}$, per i microorganismi resistenti $\mathrm{R}$, è stata valutata la MIC con E test o con il metodo della microdiluizione (20). I valori delle MIC indicano Sensibile, $\mathrm{S} \leqslant 0.06$, Intermedio, $\mathrm{I}=0.12-1$, Resistente, $\mathrm{R} \geqslant 1 \mathrm{mg} / \mathrm{L}$. Per i macrolidi è stato utilizzato il metodo Kirby-Bauer con dischetto di eritromicina $15 \mu \mathrm{g}$. Quando il ceppo è risultato resistente sono stati valutati i tre principali fenotipi Ery-R di $S$. pneumoniae. La tecnica utilizzata è stata quella del doppio disco. Sulla superficie di una piastra di Mueller-Hinton agar al $5 \%$ di sangue di montone sono stati posizionati due dischetti (eritromicina [eri] $15 \mu \mathrm{g}$ e clindamicina [cli] $2 \mu \mathrm{g}$ ) ad una distanza di 1.5-2 $\mathrm{cm}$. Dopo incubazione per 24 ore l'assenza di una zona di inibizione la crescita attorno ad entrambi i dischi è stata interpretata come espressione del fenotipo $\mathrm{C}$, mentre un alone di inibizione intorno al dischetto di clindamicina ha indicato il fenotipo M. Infine il fenotipo inducibile è stato rivelato dalla deformazione dell'alone di inibizione intorno al dischetto di clindamicina nella zona adiacente a quello di eritromicina $(25,26)$.

Eritromicino-resistenza in Streptococcus pyogenes. I tre principali fenotipi eriR di Streptococcus pyogenes sono stati identificati mediante tecnica del doppio disco come per $S$. pneumoniae.

Produzione di ESBL in E. coli e in Salmonella spp. La produzione di ESBL in E. coli è stata valutata mediante Kirby-Bauer. Quando gli aloni di inibizione sono risultati come segue ceftazidime $\leqslant 22$, aztreonam $\leqslant 27$, cefotaxime $\leqslant 27$ e ceftriaxone $\leqslant 25$, è stato utilizzato $E$ test impiegando le apposite strisce per la valutazione di questo enzima (20). In alternativa l'antibiogramma è stato ripetuto posizionando il dischetto di amoxicillina-clavulanato per 2 ore a temperatura ambiente, quindi il dischetto è stato tolto sostituendolo con quello della cefalosporina. La presenza di ESBL veniva rivelata dall'aumento dell'alone di inibizione in quest'ultimo saggio rispetto a quello eseguito con la cefalosporina da sola.

Fenotipi di ampicillino-resistenza in E. coli. Il ceppo di E. coli ampR è un isolato clinico caratterizzato fenotipicamente con il metodo Kirby-Bauer sulla base della sola resistenza all'ampicillina e sensibilità all'amoxicillinaclavulanato. Il ceppo IRT, anch'esso valutato fenotipicamente con il metodo della diffusione da dischetto, ha dimostrato resistenza sia all'ampicillina sia all'amoxicillina-clavulanato, tutti gli altri fenotipi di sensibilità o resistenza sono stati valutati sempre mediante antibiogramma.
Resistenza all'imipenem in $P$. aeruginosa. $P$. aeruginosa imiR è un ceppo di isolamento clinico identificato fenotipicamente con il metodo KirbyBauer sulla base della resistenza all'imipenem.

Metodiche utilizzate dai centri partecipanti allo studio per l'identificazione delle specie batteriche e dei loro fenotipi di resistenza.

I laboratori che hanno aderito allo studio sono stati invitati ad utilizzare le metodiche e la strumentazione abituale. Ogni centro ha successivamente specificato le metodologie usate contestualmente al risultato del test.

\section{Interpretazione dei risultati ottenuti dai centri partecipanti.}

Per i test di sensibilità agli antibiotici sono stati considerati corretti i risultati che concordavano con quelli del centro di riferimento. I risultati non corretti sono stati classificati come segue, sulla base della rilevanza sulla clinica: Errore Maggiore Grave (EMG): ceppo resistente riportato come sensibile; Errore Maggiore (EM): ceppo sensibile riportato come resistente; Errore Minore (EMi): i) ceppo resistente o sensibile riportato come intermedio, ii) ceppo intermedio riportato come sensibile o resistente.

\section{RISULTATI}

Nella tabella 3 sono riassunte le varie fasi dello studio intrapreso. I centri reclutati sono stati inizialmente 24 , scesi a 19 per il ritiro di cinque laboratori. Non tutti i centri partecipanti hanno analizzato l'intera collezione, infatti una quota di 112 microrganismi (22.6\%) risulta esclusa dall'indagine. Per quanto riguarda la valutazione della sensibilità agli antibiotici i saggi sono stati eseguiti sul $56.8 \%$ degli stipiti inviati per un totale complessivo di $281 ; 148$ saggi (29.9\%) non sono stati eseguiti Metodiche e strumentazioni utilizzate dai centri partecipanti.

Nel considerare le metodiche utilizzate dai vari centri (tabella 4), è stato osservato che il metodo semiautomatico API/ATB è stato adottato da 9 centri (47.4\%), Vitek 5 (26.3\%), Sceptor 3 (15.8\%), e Microscan 2 (10.5\%). La sensibilità agli antibiotici è stata saggiata con più metodiche oltre a quelle già segnalate per le prove di identificazione. In particolare, 5 sedi hanno utilizzato il metodo di Kirby-Bauer, 4 hanno condotto le sperimentazioni con Vitek e altrettanti con ATP/API, mentre lo Sceptor è stato impiegato da 3 centri, 2 laboratori hanno preferito il sistema Microscan ed un altro il Sensititre.

Solo 4 centri hanno citato l'uso abituale di Agar screen per l'oxacillina negli stafilococchi, l'agar screen per la vancomicina negli Enterococchi e il test del doppio disco o l'Etest per la rivelazione delle ESBL nelle Enterobacteriaceae. 
Tabella 3. Riassunto Centri Partecipanti e Ceppi analizzati

\begin{tabular}{lll} 
& Numero & \%* \\
\hline Centri reclutati & 24 & - \\
\hline Centri partecipanti & 19 & - \\
\hline Ceppi inviati ad ogni Centro & 26 & - \\
\hline Totale ceppi inviati & 494 & - \\
\hline Identificazioni eseguite & 382 & 77.3 \\
\hline Identificazioni corrette & 326 & 65.9 \\
\hline Identificazioni errate & 56 & 11.3 \\
\hline Identificazioni non eseguite & 112 & 22.6 \\
\hline Saggi di sensibilità agli antibiotici eseguiti & 346 & 70.0 \\
\hline Saggi di sensibilità agli antibiotici corretti & 284 & 57.5 \\
\hline Saggi di sensibilità agli antibiotici errati & 62 & 12.55 \\
\hline Saggi di sensibilità agli antibiotici non eseguiti & 148 & 29.9 \\
\hline ** Riferita ai 494 ceppi ricevuti in totale dai centri &
\end{tabular}

Identificazione batterica. In generale (tabella 5) è stato osservato che il metodo ATB/API e Microscan manifestano in percentuale d'errore valori molto simili (17.3 e 18.9\%), mentre i sistemi Vitek e Sceptor si attestano rispettivamente al 11.9 e $9.4 \%$. Considerando le varie specie la percentuale di identificazione corretta è risultata variabile in funzione dei patogeni considerati, tali valori sono stati calcolati sul numero di saggi effettivamente eseguiti e, precisamente corretti più errati, escludendo dal conteggio le prove non effettuate. In particolare, per quanto riguarda gli stafilococchi, i livelli di identificazione espressi in percentuale sono oscillati tra il $67.7 \%$ di $S$. aureus mecA con fenotipo inducibile al 100\% registrato con lo stesso microrganismo con fenotipo costitutivo, gli altri due isolati sono stati identificati con un'incidenza di circa $82 \%$. Gli enterococchi sono stati quelli che hanno presentato le maggiori problematiche di identificazione per tutti i centri. E. faecium, infatti, è stato ritrovato solo nel $7.1 \%$ dei casi, con E. gallinarum e E. faecium vanB riconosciuti rispettivamente dal $40 \%$ e dal $62.5 \%$ dei laboratori mentre $E$. faecalis con fenotipo amgR e vanA sono stati identificati nell'ordine da $80 \mathrm{e}$ $87.5 \%$ delle sedi.

S. pneumoniae, indipendentemente dal fenotipo di resistenza agli antibiotici posseduto, è stato correttamente identificato dall' 84 al $100 \%$ dei casi. Questi patogeni sono stati tuttavia quelli saggiati in minor misura rispetto agli altri infatti su 133 isolati da esaminare solo 88 (66.1\%) sono entrati nello studio. $S$. pyogenes è stato identificato dal 91.6 al $100 \%$ delle prove eseguite su tutti i fenotipi di resistenza. E. coli, $P$. aeruginosa e Salmonella spp sono stati riconosciuti con incidenze comprese tra $87.5 \%$ (E. coli ESBL) a $100 \%$ E. coli (cipR), P. aeruginosa (imiR) e Salmonella spp delle prove eseguite. Globalmente su 382 identificazioni effettuate 326 (85.4\%) sono risultate corrette.
Prove di Sensibilità. La tabella 6 riassume i risultati ottenuti sui diversi microrganismi. Da notare, solo 7 Laboratori (36.8\%) hanno esaminato l'intera collezione, 4 centri $(21 \%)$ hanno eseguito i test sulla metà dei ceppi inviati. Globalmente i saggi non eseguiti variano in percentuale dal $10.5 \%$ (S. aureus mecA I e penR) al $42 \%$ (S. pneumoniae penR e eriS). Per quanto riguarda il tipo di errore, il $63 \%$ di E. coli IRT, $48 \%$ di S. haemolyticus TeiR, $47 \%$ di E. coli ESBL sono stati errati completamente (EMG). Rientrano nella stessa categoria di prova non corretta E. faecium ampR (42\%), E. faecalis VanA $(37 \%)$ e in minor misura gli altri isolati (tabella 6). Errore grave (EM) è stato ritrovato con $S$. haemolyticus teiR(26\%), E. faecalis amgR (21\%); S. pneumoniae e $S$. pyogens eriS (5\%). Saggi non corretti ma di categoria Emi hanno coinvolto $S$. pneumoniae penS e penI $(21 \%), E$. gallinarum VanC, $S$. pneumoniae penR, e $S$. pyogenes eriR e con minor incidenza gli altri ceppi.

Per quanto concerne la valutazione della sensibilità agli antibiotici riferita al sistema adottato (tabella 7), le percentuali di errore sono oscillate tra 11.9 di ATB/API e $28.1 \%$ di Microscan, mentre sulle 346 prove di sensibilità eseguite l'errore è stato $17.9 \%$. Prendendo in esame gli stafilococchi, le maggiori difficoltà sono state registrate con i ceppi produttori di penicillinasi, quelli resistenti alla tecoplanina e $\mathrm{i}$ meticillino-resistenti con fenotipo inducibile che hanno dimostrato valori di riconoscimento dell'insensibilità di $47.0, \quad 53.3$ e $58.8 \%$ rispettivamente. Più alta è stata la frequenza con la quale è stato identificato il fenotipo mecA costitutivo fissata a $81.2 \%$ delle valutazioni totali. Negli enterococchi l'ampicillino-resistenza è stata ritrovata solo nel $42.8 \%$ dei casi, gli altri caratteri di resistenza sono stati accertati con incidenza dal $84.6 \%$ (vanC) al $100 \%$ (vanB). I diversi fenotipi di insensibilità alla penicillina e all'eritromicina in $S$. pneumoniae sono stati individuati con frequenze comprese tra $60 \%$ di PEN-S al $92.3 \%$ di eriR M, anche in questo caso il numero dei ceppi saggiati è stato di $82(61.6 \%)$ un valore piuttosto ridotto rispetto al numero di ceppi inviati (133). Considerando S. pyogenes tutti i caratteri di resistenza sono stati trovati con incidenze variabili tra 78.6 (eriR C) e 92.3 (eriR M). Tutti i fenotipi di refrattarietà dei Gramnegativi e cioè ESBL, IRT, ampR, cipR e imiR sono stati tutti accertati correttamente $(100 \%)$ con l'eccezione di ESBL in Salmonella spp. riconosciuta solo nel $69.2 \%$ dei saggi. In totale l'accertamento corretto delle resistenze nei ceppi studiati è stato del $82.8 \%$. 
Tabella 4. Metodi utilizzati dai Centri per l'identificazione e per i saggi di sensibilità agli antibiotici dei 494 ceppi inviati

\begin{tabular}{lllllllll}
\hline & \multicolumn{2}{l}{ IDENTIFICAZIONE DEI CEPPI (N.) } & \multicolumn{4}{l}{ SAGGI DI SENSIBILITÀ AGLI ANTIBIOTICI (N.) } \\
\hline Metodo di identificazione & Centri (\%) & Corrette & Errate & Non eseguite & Centri (\%) & Corretti & Errati & Non eseguiti \\
\hline ATB/API & $9(47.4)$ & $I 48$ & $3 I$ & 69 & $4(2 I . I)$ & 59 & 8 & 37 \\
\hline Vitek & $5(26.3)$ & $8 I$ & $I I$ & 17 & $4(2 I . I)$ & 67 & 14 & 24 \\
\hline Sceptor & $3(15.8)$ & 67 & 7 & 7 & $3(15.8)$ & 54 & 16 & 7 \\
\hline Microscan & $2(10.5)$ & 30 & 7 & 19 & $2(10.5)$ & 23 & 9 & 20 \\
\hline Kirby-Bauer & - & - & - & - & $5(26.3)$ & 59 & 11 & 60 \\
\hline Sensititre & - & - & - & - & $1(5.2)$ & 22 & 4 & 0 \\
\hline TOTALE & 19 & 326 & 56 & 112 & 19 & 284 & 62 & 148 \\
\hline
\end{tabular}

Tabella 5. Metodi utilizzati per l'identificazione della specie batterica.

\begin{tabular}{|c|c|c|c|c|c|c|c|c|c|c|c|c|c|}
\hline & & \multicolumn{2}{|c|}{ ATB/API } & \multicolumn{3}{|c|}{ MICROSCAN } & \multicolumn{2}{|c|}{ SCEPTOR } & \multicolumn{2}{|l|}{ VITEK } & \multicolumn{3}{|c|}{ TOTALE } \\
\hline & & $\mathrm{C}$ & $\mathrm{E}$ & $\mathrm{C}$ & $\mathrm{E}$ & C & $\mathrm{E}$ & C & $\mathrm{E}$ & $\mathrm{C}$ & $E$ & n.e. & $\% \mathrm{C}$ \\
\hline I & S. aureus mecA C & 9 & - & 2 & - & 3 & - & 4 & - & 18 & - & 1 & 100.0 \\
\hline 2 & S. aureus mecA I & 5 & 4 & - & $\mathrm{I}$ & 3 & - & 3 & $\mathrm{I}$ & 11 & 6 & 2 & 67.7 \\
\hline 3 & S. aureus Penicillasi+ & 8 & I & - & I & 2 & I & 4 & - & 14 & 3 & 2 & 82.3 \\
\hline 4 & S. haemolyticus tei $\mathrm{R}$ & 9 & - & - & $\mathrm{I}$ & 2 & $\mathrm{I}$ & 2 & $\mathrm{I}$ & 13 & 3 & 3 & 81.2 \\
\hline 5 & E. faecalis VanA & 7 & $\mathrm{I}$ & $\mathrm{I}$ & $\mathrm{I}$ & 3 & - & 3 & - & 14 & 2 & 3 & 87.5 \\
\hline 6 & E. faecium VanB & 4 & 5 & $\mathrm{I}$ & - & 2 & - & 3 & I & 10 & 6 & 3 & 62.5 \\
\hline 7 & E. gallinarum VanC & 2 & 6 & - & $\mathrm{I}$ & 3 & - & $\mathrm{I}$ & 2 & 6 & 9 & 4 & 40.0 \\
\hline 8 & E. faecalis amgR & 6 & I & $\mathrm{I}$ & I & 3 & - & 2 & I & 12 & 3 & 4 & 80.0 \\
\hline & E. faecium ampR & - & 7 & - & $\mathrm{I}$ & - & 3 & I & 2 & $\mathrm{I}$ & 13 & 5 & 7.1 \\
\hline & S. pneumoniae penS & 5 & $\mathrm{I}$ & $\mathrm{I}$ & - & 2 & - & 3 & - & 11 & $\mathrm{I}$ & 7 & 91.6 \\
\hline & S. pneumoniae penl & 5 & - & 2 & - & 3 & - & 3 & - & 13 & - & 6 & 100.0 \\
\hline & S. pneumoniae pen R & 4 & $\mathrm{I}$ & 2 & - & 3 & - & 3 & - & 12 & $\mathrm{I}$ & 6 & 92.3 \\
\hline & S. pneumoniae eriR C & 3 & 2 & 2 & - & 3 & - & 3 & - & II & 2 & 6 & 84.6 \\
\hline & S. pneumoniae eriR M & 6 & - & 2 & - & 3 & - & 3 & - & 14 & - & 5 & 100.0 \\
\hline & S. pneumoniae eriR I & 5 & $\mathrm{I}$ & 2 & - & 3 & - & 3 & - & 13 & $\mathrm{I}$ & 5 & 92.8 \\
\hline & S. pneumoniae eriS & 5 & - & I & - & I & - & 3 & - & 10 & - & 9 & 100.0 \\
\hline & S. pyogenes eriS & 4 & $\mathrm{I}$ & $\mathrm{I}$ & - & 3 & - & 3 & - & II & $\mathrm{I}$ & 7 & 91.6 \\
\hline & S. pyogenes eriR C & 7 & - & 2 & - & 3 & - & 2 & I & 14 & I & 4 & 93.3 \\
\hline & S. pyogenes eriR M & 6 & - & 2 & - & 3 & - & 2 & - & 13 & - & 6 & 100.0 \\
\hline & S. pyogenes eriR I & 6 & - & $\mathrm{I}$ & - & 3 & - & 2 & $\mathrm{I}$ & 12 & $\mathrm{I}$ & 6 & 92.3 \\
\hline & E. coli ESBL & 7 & - & I & - & 2 & $\mathrm{I}$ & 4 & I & 14 & 2 & 3 & 87.5 \\
\hline & E. coli cipR & 7 & - & $\mathrm{I}$ & - & 3 & - & 5 & - & 16 & - & 3 & 100.0 \\
\hline & E. coli ampR & 7 & - & $\mathrm{I}$ & - & 2 & $\mathrm{I}$ & 4 & - & 14 & $\mathrm{I}$ & 4 & 93.3 \\
\hline 24 & E. coli IRT & 7 & - & I & - & 3 & - & 5 & - & 16 & - & 3 & 100.0 \\
\hline & P. aeruginosa imiR & 7 & - & 2 & - & 3 & - & 5 & - & 17 & - & 2 & 100.0 \\
\hline \multicolumn{14}{|c|}{26 Salmonella } \\
\hline & spp. TEM25/CTX2 & 7 & - & 1 & - & 3 & - & 5 & - & 16 & - & 3 & 100.0 \\
\hline & cale parziale & 148 & 31 & 30 & 7 & 67 & 7 & 81 & II & 326 & 56 & 112 & 85.4 \\
\hline & cale complessivo (corr+err) & 179 & & 37 & & 74 & & 92 & & 382 & & & \\
\hline & centuale & & 17.3 & & 18.9 & & 9.4 & & 11.9 & & 14.6 & & \\
\hline
\end{tabular}

C, corretto; E, errato; n.e., non eseguito.

\section{DISCUSSIONE}

I dati raccolti in questo studio possono essere così riassunti: 19 laboratori di Microbiologia clinica, di cui 3 privati, distribuiti sul territorio ligure con l'inclusione di 2 centri del basso Piemonte hanno analizzato tutti i 26 microrganismi veicolanti meccanismi di resistenza agli antibiotici ben caratterizzati. Solo 7 Laboratori hanno esaminato l'intera collezione di 26 isolati inviata, mentre complessivamente 12 sedi operative hanno studiato almeno 20 ceppi. Nel loro insieme i centri hanno valutato correttamente $85.4 \%$ delle prove di identificazioni eseguite e $82.8 \%$ dei saggi per la sensibilità agli antibiotici effettuati. Il riconoscimento delle specie batteriche ha presentato maggiori difficoltà con gli enterococchi, in particolare con E. faecium, mentre più facile è sembrata l'dentificazione delle specie batteriche Gram-negative. In generale salvo qualche eccezione i patogeni inviati hanno creato qualche difficoltà per il loro riconoscimento come specie batterica a molti laboratori. Lo stesso dicasi per l'accertamento delle resistenze agli antibiotici, con problematiche più evidenti per stafilococchi ed enterococchi. Su tutte la statistiche pesa il numero dei ceppi non esaminati che hanno rappresentato il $22.6 \%$ delle identificazioni e il $29.9 \%$ delle prove di sensibilità agli antibiotici. Alcuni dei ceppi utilizzati per lo studio, infine, presentavano fenotipi di resistenza piuttosto rari: se, tuttavia, non sono identificati correttamente possono dar 
Tabella 6. Riassunto risultati prove di sensibilità agli antibiotici

\begin{tabular}{|c|c|c|c|c|c|c|}
\hline \multirow[t]{2}{*}{ Ceppo } & \multirow[t]{2}{*}{ Fenotipo di Resistenza } & \multirow[t]{2}{*}{$\begin{array}{c}\text { Test non eseguiti } \\
(\%)\end{array}$} & \multirow[t]{2}{*}{$\begin{array}{l}\text { Test corretti } \\
(\%)\end{array}$} & \multicolumn{3}{|c|}{$\begin{array}{c}\text { Test non corretti } \\
\text { (\%) }\end{array}$} \\
\hline & & & & EMG* & $\mathrm{EM}^{* *}$ & EMi*** \\
\hline S. aureus mecA costitutivo & oxaR & 21 & 68 & 10 & & \\
\hline S. aureus mecA inducibile & oxaR & 10.5 & 53 & 37 & & \\
\hline S. aureus penicillasi+ & b-lattamasi & 10.5 & 32 & 48 & & \\
\hline S. haemolyticus teiR & Teicoplanino-R & 21 & 16 & 37 & 26 & \\
\hline E. faecalis VanA & Vancomicino-R & 26 & 68 & 5 & & \\
\hline E. faecium VanB & Vancomicino-R & 32 & 68 & & & \\
\hline E. gallinarum Van $C$ & Vancomicino-R & 32 & 37 & & 21 & 10 \\
\hline E. faecalis amgR & Aminoglicosidi-R alti livelli & 37 & 63 & & & \\
\hline E. faecium ampR & Ampicillino- $R$ & 21 & 32 & 42 & & \\
\hline S. pneumoniae penS & Penicillino-S & 42 & 32 & & & 21 \\
\hline S. pneumoniae penl & Penicillino-R & 42 & 32 & & & 21 \\
\hline S. pneumoniae penR & Penicillino-R & 42 & 42 & 5 & & 10 \\
\hline S. pneumoniae eriR costitutivo & Eritromicino-R & 37 & 58 & 5 & & \\
\hline S. pneumoniae eriR fenotipo $M$ & Eritromicino-R & 32 & 63 & 5 & & \\
\hline S. pneumoniae eriR inducibile & Eritromicino-R & 37 & 53 & 10 & & \\
\hline S. pneumoniae eriS & Eritromicino-S & 42 & 53 & & 5 & \\
\hline S. pyogenes eriS & Eritromicino-S & 5 & 42 & & 5 & \\
\hline S. pyogenes eriR costitutivo & Eritromicino-R & 26 & 5 & 16 & & 5 \\
\hline S. pyogenes eriR fenotipo $M$ & Eritromicino-R & 26 & 57 & 5 & & 10 \\
\hline S. pyogenes eriR inducibile & Eritromicino-R & 31 & 5 & 10 & & 5 \\
\hline E. coli ESBL & ESBL & 21 & 32 & 47 & & \\
\hline E. coli cipR & Ciprofloxacino-R & 21 & 79 & & & \\
\hline E. coli ampR & Ampicillino- $R$ & 26 & 74 & & & \\
\hline E. coli IRT & Amoxicillina-clavulanato- $R$ & 21 & 16 & 63 & & \\
\hline$P$. aeruginosa imiR & Imipenem-R & 26 & 68 & 5 & & \\
\hline Salmonella spp ESBL & ESBL & 26 & 68 & 5 & & \\
\hline
\end{tabular}

* EMG= Errore Maggiore Grave; **EM= Errore Maggiore; ***EMi =Errore Minore

Tabella 7. Metodi utilizzati per la valutazione della sensibilità agli antibiotici

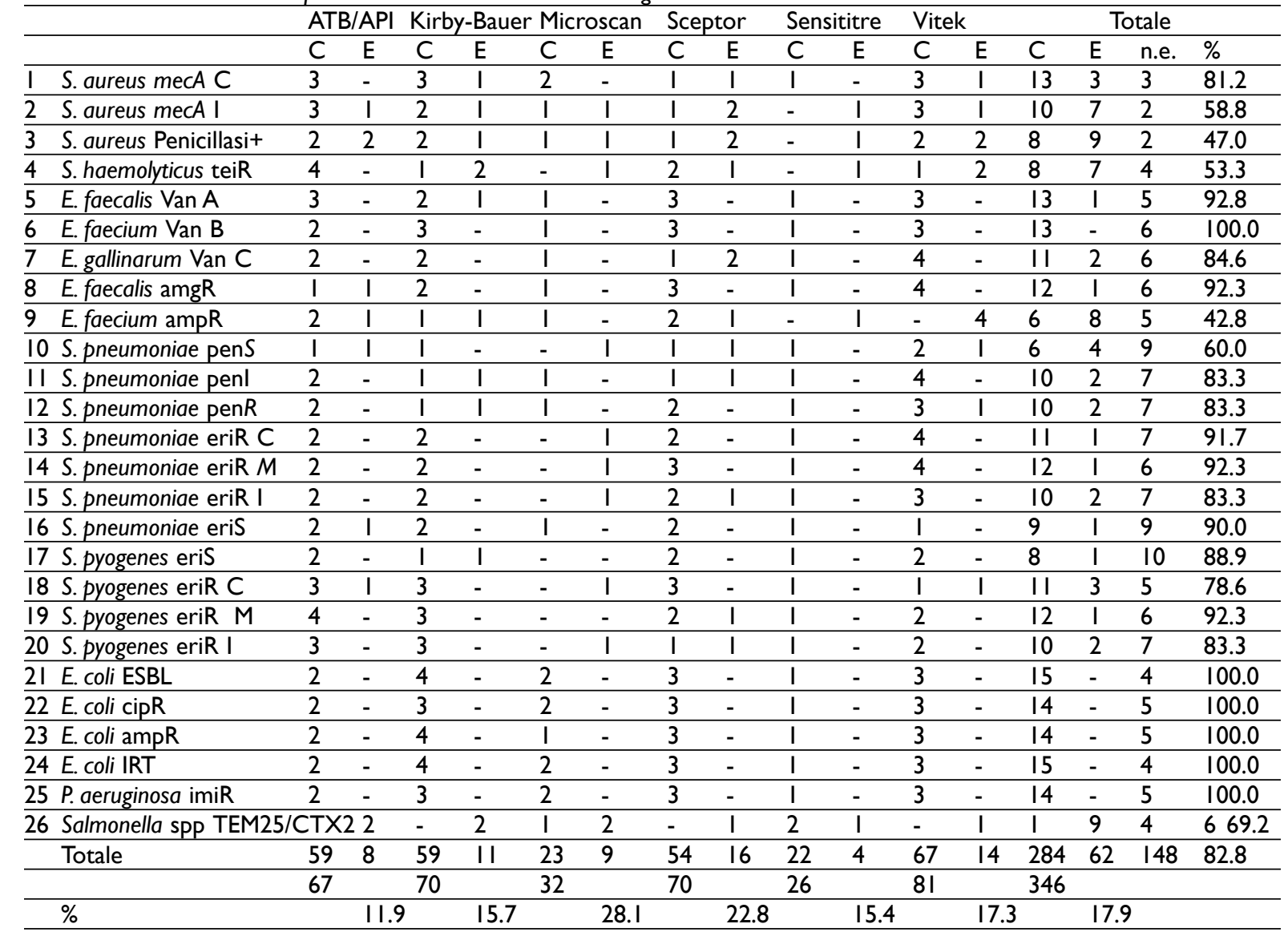

C, corretto; $E$, errato; n.e., non eseguito. 
luogo a problemi non solo dal punto di vista terapeutico ma anche economico $(11,21,23,28$, 29).

Questi dati consentono alcune considerazioni di carattere più generale. Un primo problema, indipendentemente dalla strumentazione automatica o semiautomatica adottata che ha certo i suoi limiti ben noti all'operatore $(7,8)$, riguarda la mancanza di uniformità dei metodi di saggio, che assai raramente si rifà a linee guida riconosciute a livello nazionale e internazionale. I metodi di conferma dei risultati prodotti dalla strumentazione sono infatti scarsamente usati.

Il fatto di non poter diversificare i saggi sulla base del tipo di combinazione patogeno/antibiotico, dovute talvolta a carenze di personale qualificato appare come fattore condizionante l'esito dell'esame batteriologico. Un saggio errato ha una ricaduta negativa immediata per il paziente $(21,28)$ ed in seconda istanza determina decorsi terapeutici più lunghi e perciò più costosi $(21,27$, 28). Quando non esistono le condizioni ottimali di esecuzione di un esame microbiologico, sulla base delle attuali conoscenze, sia dal punto di vista dell'identificazione corretta della specie batterica (14), sia da quello dell'accertamento delle resistenze, sarebbe preferibile non eseguire quel tipo di esame di laboratorio deleteria per la terapia $(21,28)$.

Il collegamento con un Centro di riferimento e la creazione di una rete di scambi collaborativi tra le varie realtà potrebbe risolvere facilmente queste problematiche.

Questo studio ha messo chiaramente in luce come tale esigenza debba essere sentita da una parte dei centri coinvolti in questa indagine. All'entusiasmo dell'adesione non ha fatto seguito una risposta adeguata ma l'analisi dei propri risultati, confrontata con le indicazioni metodologiche fornite dal Centro di riferimento, fa ben pensare a migliori risultati nelle iniziative future.

Questa prima iniziativa ha avuto certamente il suo primo importante successo grazie ai citati scambi di informazione che hanno permesso quasi automaticamente di stilare una sorta di linee guida, qui riassunte in parte nella Sezione Materiali e Metodi, sulla quale cercare di uniformare la diagnostica col fine di renderla aggiornata e tecnicamente corretta. Ogni altra via risulterà estremamente onerosa per il paziente, per le strutture sanitarie e in definitiva per la Società $(21,28)$.

Un altro aspetto importante riguarda il mancato inserimento, da parte dei centri, dei ceppi ATCC inviati per il controllo di qualità, nei saggi eseguiti (20). Anche questo fattore può essere preso come indice di una mancanza di un riferimento e di linee guida ben collaudate adottate dal laboratorio per condurre la routine (20).

\section{RINGRAZIAMENTI}

Gli autori sono indebitati con Elisabetta Maioli per l'aiuto fornito durante alcune fasi di questo lavoro.

\section{BIBLIOGRAFIA}

1. Acar JF. Consequences of bacterial resistance to antibiotics in medical practice. Clin Infect Dis 1997, 24 (Suppl. 1): S17-18.

2. Boisivon A, Thibault M, Leclercq R. Colonization by vancomycin-resistant enterococci of the intestinal tract of patients in intensive care units from French general hospitals. Clin Microbiol Infect 1997; 3: 175-9.

4. Chelossi E, Platè M, DeLeo C, Schito GC. Analisi genotipica di Streptococcus pyogenes eritromicinoresistenti isolati recentemente in Italia GIMMOC 1998; 2: 9-20.

5. Chelossi E, Platè M, De Leo C, Schito GC. Attività di alcuni antibiotici su Streptococcus pyogenes eritromicino-resistenti isolati recentemente in Italia. GIMMOC 1997; 3: 9-20.

6. Chung $\mathrm{M}$, de Lencastre $\mathrm{H}$, Matthews $\mathrm{P}$, et al. Molecular typing of methicillin-resistant Staphylococcus aureus by pulsed-field gel electrophoresis: comparison of results obtained in a multilaboratory effort using identical protocols and MRSA strains. Microb Drug Resist 2000; 6: 189-98.

7. Davies J. Inactivation of antibiotics and dissemination of resistance genes. Science 1994; 264: 375-82.

8. Felmingham D, Brown DFJ. Instrumentation in antimicrbial susceptibility testing. J Antimicrob Chemother 2001; 48 (suppl S1), 81-5.

9. Ferraro MJ, Jorgensen JH. Susceptibility testing instrumentation and computerized expert systems for data analysis and interpretation. Murray PR et al.: Manual of Clinical Microbiology, Seventh Edition, p. 1593-1600, ASM Press. 1999.

10. Gold HS, Moellering RC. Antimicrobial-drug resistance. N Engl J Med 1996, 35: 1445-53.

11. Jones RN, Masterson R. Determining the value of antimicrobial surveillance programs. Diagn Microbiol Infect Dis 2001; 41: 171-5.

12. Kahlmeter G, Brown DFJ. Resistance surveillance studies comparability of results and quality assurance methods. J Antimicrob Chemother 2002; 50: 775-7.

13. Levy SB. Multidrug resistance - A sign of the times. N Engl J Med 1998; 338: 1376-8.

14. Livermore D, Struelens M, Amorin J, et al. Multicentre evaluation of the VITEK 2 advanced export system for interpretative reading of antimicrobial resistance tests. J Antimicrob Chemother 2002; 49: 289-300.

15. Livermore DM, Winstanley TG, Shannon KP. Interpretative reading: recognizing the unusual and inferring resistance mechanisms from resistance phenotyoes. J Antimicrob Chemother 2001; 48, Suppl S1: 87-102.

16. Marchese A, Debbia EA, Bacca D, Balistreri G, Musolino B, Schito GC. Multidrug-resistant Grampositive pathogens: an update on current 
microbiological patterns. Drugs 1997; 54: Suppl. 6, 11-20.

17. Marchese A, Saverino D, Debbia EA, Pesce A, Schito GC. Antistaphylococcal activity of cefdinir, a new oral third generation cephalosporin, alone and in combination with other antibiotics, at supra- and subMIC levels. J Antimicrob Chemother 1995; 35: 53-66.

18. Marchese A, Arlet G, Schito GC, Lagrange PH, Philippon A. Detection of SHV-5 extended-spectrum beta-lactamase in Klebsiella pneumoniae strains isolated in Italy. Eur J Clin Microbiol Infect Dis 1996; 15: 245-8.

19. Marchese A, Tonoli E, Balistreri G, Debbia EA, Schito GC. Antibiotic susceptibility patterns and serotypes of antibiotic resistant and or invasive Streptococcus pneumoniae strains circulating in Italy. Microbial Drug Res 2000; 6: 163-70.

20. Marchese A, Tonoli E, Debbia EA, Schito GC. Macrolide resistance mechanisms and expression of phenotypes among Streptococcus pneumoniae circulating in Italy. J Antimicrob Chemother 1999; 44: 461-4

21. National Committee for Clinical Laboratory Standards. Performance standards for antimicrobial susceptibility testing. Twelfth informational supplement: M100-S12, Vol. 22. NCCLS Wayne, PA. 2002.

22. Nicolau D. Clinical and economic implications of antimicrobial resistance for the management of community-acquired respiratory tract infections. J Antimicrob Chemother 2002; 50 (Suppl S1): 61-70.

23. O'Brien TF. The global epidemic nature of antimicrobial resistance and the need to monitor and manage it locally. Clin Infect Dis 1997; 24 Suppl 1: S2-S8.

24. O'Brien TF. Emergence, spread and environmental effect of antimicrobial resistance: how use of an antimicrobial anywhere can increase resistance to any antimicrobial anywhere else. Clin Infect Dis 2002; 34 (Suppl 3) S78-84.

25. Schito GC, Molinari G, Debbia EA, et al. Mechanisms of aminoglycoside resistance in a large italian hospital. Abstr A-67, 90th Annual Meeting ASM, 1990.

26. Seppälä H, Nissinen A, Yu Q, Huovinen P. Three different phenotypes of erythromycin-resistant Streptococcus pyogenes in Finland. J Antimicrob Chemother 1993; 32: 885-91.

27. Sutcliffe J, Tait-kamradt A, Wondrack L. Streptococcus pneumoniae and Streptococcus pyogenes resistant to macrolides but sensitive to clindamycin: a common resistance pattern mediated by an efflux system. Antimicrob Agents Chemoter 1996; 40: 1817-24.

28. Tenover FC. Development and spread of bacterial resistance to antimicrobial agents: an overview. Clin Infec Dis 2001; (Suppl 3): S108-15.

29. Travers K, Barza M. Morbidity of infections caused by antimicrobial-resistant bacteria. Clin Infect Dis 2002, 34: (Suppl 3): S131-34.

30. Wood MJ and R.C. Moellering. Microbial resistance: bacteria and more. Clin Infect Dis 2003; 36 (suppl 1): S2-3.

\section{Eugenio A. Debbia}

Istituto di Microbiologia,

Università di Genova

Facoltà di Medicina e Chirurgia,

Largo Rosanna Benzi 10, - 16132 Genova

Tel. +39-10-3537655; Fax +39-10-504837

E-mail: eugenio.debbia@unige.it 